\title{
Is fever control or improved survival the 'risk factor' for ventilator-associated pneumonia?
}

\author{
Matthew BA Harmon ${ }^{1 *}$, Theis $S$ Itenov $^{2}$, Jens $U$ Jensen ${ }^{3}$ and Nicole P Juffermans ${ }^{1}$ \\ See related research by Launey et al. http://ccforum.com/content/18/6/689
}

We read with interest the paper by Launey and colleagues [1] regarding the effect of fever control on the incidence of ventilator-associated pneumonia (VAP) in brain-injured patients. We commend the authors for addressing this relationship, given that fever and nosocomial infections are frequent in these patients. However, we have concerns regarding the interpretation of data.

Firstly, independent risk factors for VAP, namely disease severity and lung contusion [2,3], are unevenly distributed between groups. In addition, other risk factors, such as chronic obstructive pulmonary disease (COPD) and ICU readmission $[2,4]$, were not taken into account, suggesting any effect of fever control is likely subject to confounding.

Secondly, mortality in the control group was higher (34\% versus $23 \%$ ), lowering the observation period if death occurred within 28 days. Thus, patients in the fever-control group were observed for a longer period of time, increasing their time at risk for VAP.

Thirdly, VAP incidence was higher in patients who were subject to fever control for longer than 3 days. However, duration of fever control was likely determined by factors also affecting the patients' risk of acquiring VAP; that is, death. Not surprisingly, those who died within 3 or fewer days of initiating fever control did not develop VAP as frequently as those who did not.

In summary, the intervention group and historical control group in this study do not seem to be optimally matched on crucial parameters. Additionally, decreased mortality and longer follow-up in the intervention group likely resulted in uncontrolled lead time bias/attrition bias. Decreased mortality also seems contradictory to the claim that VAP, a deadly condition for ICU patients, is more frequent among fever-control patients.

\section{Authors' response}

Yoann Launey, Nicolas Nesseler, Fanny Feuillet, Yannick Mallédant and Philippe Seguin

We thanks Dr Harmon and colleagues for their comments, which we would like to nuance.

Indeed, COPD was not reported at admission; however, such disease is clearly unusual in the studied population. In a recent multicentre study performed in the same population, a history of respiratory disease (including COPD) was observed in only 3.6\% [5]. Secondly, none of the included patients were readmitted in our ICU during the first 28 days of ICU stay, which was the endpoint period to assess VAP occurrence.

As reported in the statistical section, we used a competing risk multivariate model [6] which considers death within 28 days as a competing event. Consequently, this model takes into account only the periods of time during which the patients were exposed to the risk of VAP. Moreover, the benefit of this model is that the death event is not a confounding factor in the determination of VAP incidence between the patients receiving fever control for longer than 3 days and those who did not. The size of the study was not large enough to reach statistical significance.

Finally, we acknowledge that the matching of the two groups was not optimal in our observational study and obviously a prospective randomized study is required.

* Correspondence: m.harmon@amc.uva.nl

${ }^{1}$ Department of Intensive Care Medicine, Laboratory of Experimental Intensive Care and Anesthesiology, Academic Medical Center, University of Amsterdam, Meibergdreef 9, Amsterdam 1105 AZ, The Netherlands Full list of author information is available at the end of the article 
Nevertheless, we would like to emphasize our study was not designed to assess the impact of fever-control strategy on mortality and, despite the apparent results, no conclusion can be drawn on mortality. Moreover, a recent meta-analysis showed VAP was not associated with over-mortality in medical and trauma patients [7].

\section{Abbreviations}

COPD: Chronic obstructive pulmonary disease; VAP: Ventilator-associated pneumonia.

\section{Competing interests}

The authors declare no competing financial interests. The authors are investigators (MBAH, NPJ), coordinator (TSI) and study director (JUJ) on a study of mild-induced hypothermia in severe sepsis and septic shock.

\section{Authors' contributions}

MBAH and TSI drafted the letter. JUJ and NPJ critically revised for important intellectual content. All authors read and approved the final manuscript.

\section{Author details}

${ }^{1}$ Department of Intensive Care Medicine, Laboratory of Experimental Intensive Care and Anesthesiology, Academic Medical Center, University of Amsterdam, Meibergdreef 9, Amsterdam 1105 AZ, The Netherlands. ${ }^{2}$ Nordsjællands Hospital, Department of Anesthesiology, Hillerød, Denmark. ${ }^{3}$ Department of Rheumatology and Infectious Diseases, Rigshospitalet, University of Copenhagen, CHIP, Finsen Centret, Copenhagen, Denmark.

\section{Published online: 30 April 2015}

\section{References}

1. Launey Y, Nesseler N, Le Cousin A, Feuillet F, Garlantezec R, Malledant Y. Effect of a fever control protocol-based strategy on ventilator-associated pneumonia in severely brain-injured patients. Crit Care. 2014;18:689.

2. Tejerina E, Frutos-Vivar F, Restrepo Ml, Anzueto A, Abroug F, Palizas F, et al. Incidence, risk factors, and outcome of ventilator-associated pneumonia. J Crit Care. 2006;21:56-65.

3. Hanes SD, Demirkan K, Tolley E, Boucher BA, Croce MA, Wood GC, et al. Risk factors for late-onset nosocomial pneumonia caused by Stenotrophomonas maltophilia in critically ill trauma patients. Clin Infect Dis. 2002;35:228-35.

4. Christmas AB, Freeman E, Chisolm A, Fischer PE, Sachdev G, Jacobs DG, et al. Trauma intensive care unit 'bouncebacks': identifying risk factors for unexpected return admission to the intensive care unit. Am Surg. 2014;80:778-82.

5. Seguin $\mathrm{P}$, Laviolle B, Dahyot-Fizelier $C$, Dumont $R$, Veber B, Gergaud S, et al. Effect of oropharyngeal povidone-iodine preventive oral care on ventilator-associated pneumonia in severely brain-injured or cerebral hemorrhage patients: a multicenter, randomized controlled trial. Crit Care Med. 2014;42:1-8.

6. Fine J, Gray R. A proportional hazards model for the subdistribution of a competing risk. J Am Stat Assoc. 1999;94:496-509.

7. Melsen WG, Rovers MM, Groenwold RHH, Bergmans DCJJ, Camus C, Bauer

T, et al. Attributable mortality of ventilator-associated pneumonia: a meta-analysis of individual patient data from randomised prevention studies. Lancet Infect Dis. 2013;13:665-71. 